\title{
Product Scent and Memory
}

\author{
ARADHNA KRISHNA \\ MAY O. LWIN \\ MAUREEN MORRIN
}

\begin{abstract}
Scent research has focused primarily on the effects of ambient scent on consumer evaluations. We focus instead on the effects of product scent on consumer memories. For instance, if a pencil or a facial tissue is imbued with scent (vs. not), recall for the brand's other attributes increases significantly-with the effects lasting as much as 2 weeks after exposure. We also find that product scent is more effective than ambient scent at enhancing memory for product information. We suggest that this may be because, with product (ambient) scent, scent-related associations are focused on a single object (are diffused across multiple objects) in the environment. In support, we find that the memory effects are driven by the number of product/scent-related associations stored in long-term memory. The results suggest that, although ambient scent has received the bulk of attention from researchers and managers in recent years, greater focus on product scent is warranted.
\end{abstract}

$\mathrm{T}_{\mathrm{r}}$ he actions of firms in recent years suggest that imbuing products with scents can enhance brand equity. Firms have sought scent-based trademarks for products such as sewing thread (Brookman 1999), automobile tires (Carter 1995), and tennis balls (Bird 2000). Are such trademark protection efforts worthwhile? We attempt to answer this question by exploring the effect of product scent on consumers' ability to remember product information over time.

To date, consumer research on scent has focused primarily on the effects of scent on product evaluation (Bone and Ellen 1999; Bone and Jantrania 1992; Bosmans 2006; Laird 1932; Mitchell, Kahn, and Knasko 1995; Spangenberg, Crowley, and Henderson 1996) as well as lingering time (Spangenberg et al. 1996) and variety-seeking behavior

\footnotetext{
Aradhna Krishna is the Dwight F. Benton Professor of Marketing at the Ross School of Business, University of Michigan, 701 Tappan Street, Ann Arbor, MI 48109-1234 (aradhna@umich.edu). May O. Lwin is associate professor, Wee Kim Wee School of Communication and Information, Nanyang Technological University, 31 Nanyang Link, Singapore 637718 (tmaylwin@ntu.edu.sg). Maureen Morrin is professor of marketing, Rutgers University School of Business, 227, Penn St., Camden, NJ 08102 (mmorrin @ rutgers.edu). Address correspondence to Aradhna Krishna. The authors are listed in alphabetical order and contributed equally to the research. The authors would like to thank Mindawati Wijaya and Raslyn Rasiah for their help in data collection, as well as the editor, the associate editor, and the reviewers for helpful input. The authors would also like to acknowledge financial support from internal research grants received from the University of Michigan and Rutgers University in support of the project.
}

John Deighton served as editor and Laura Peracchio served as associate editor for this article.

Electronically published December 4, 2009
(Mitchell et al. 1995). Some initial work has examined the effects of scent on memory (Morrin and Ratneshwar 2000, 2003), but these efforts have focused exclusively on the effects of ambient scent rather than product scent. Yet the use of product scent would appear to be much more widespread in the marketplace. Moreover, product scent may be particularly effective at enhancing memory for product information as a function of its ability to enhance a product's distinctiveness within its surrounding context.

Specifically, we show that, when products are scented (vs. not), consumers are more likely to remember information about those products. This occurs even though the product scent is not reintroduced at the time of recall and even when memory is assessed as much as 2 weeks after product exposure. In exploring the process at work for this effect, we show that the number of scent-related thoughts available at the time of information retrieval mediates the effect of the product scent on memory for product information. Additionally, ambient scent is not found to exhibit a similar effect on memory for an individual product, and we argue that this is due to a product scent's ability to make a product perceptually distinctive within its environment. We rule out alternative explanations for a product scent's effect on memory, namely, the amount of cognitive processing, the number of scent-related thoughts during product exposure, the amount of pleasure and arousal associated with the product, product evaluation, and time spent with the product.

The rest of this article is organized as follows. First, we present a literature review on scent research and offer a conceptual framework. Then we present the results of two 
studies that begin to empirically explore these issues. We conclude with implications and limitations of the research.

\section{LITERATURE REVIEW AND CONCEPTUAL FRAMEWORK}

Research on scent pertinent to our work has been conducted primarily by consumer behavior researchers and cognitive psychologists. Figure 1 shows the relationship between the various constructs in studies across these different research streams, and it also includes what we propose, that is, our conceptual framework. Thus, figure 1 includes our earlier mention of consumer research on the effect of product scent on product evaluation and so forth. We now discuss in greater detail prior research that is pertinent to our focus on scent and memory.

\section{Scent Recognition and Scent Memory}

In the area of psychology, most of the research on the relationship between scent and memory has tested people's abilities to recognize the scent itself rather than their ability to remember information associated with a scent (Engen and Ross 1973; Zucco 2003). Much of this research focuses on forgetting rates for information (Engen, Kuisma, and Eimas 1973; Pe- terson and Peterson 1959; Shepard 1967). For example, when researchers study the recall of, say, verbal information over time, they typically find that rapid forgetting occurs, with the most rapid decline in memory occurring immediately after learning (Peterson and Peterson 1959). In comparison, odor recognition studies have shown that the ability to recognize scents previously smelled decays very little over time, with minimal reductions in recognition accuracy from seconds (Engen et al. 1973) to months after exposure (Engen and Ross 1973; Zucco 2003). Engen and Ross (1973) found that scent recognition was $70 \%$ when tested immediately after exposure versus $65 \%$ after a 1 -year delay, as compared to picture recognition (Shepard 1967), which was 99\% when measured immediately after exposure versus $58 \%$ after a 4 -month delay.

Recent research in the basic sciences suggests that there may be physiological and/or neurological reasons for our ability to remember scents so well over time (Buck and Axel 1991). However, the precise mechanism underlying the persistence of scent recognition memory has not been established. Nevertheless, the empirical results suggest that, if product scent does enhance consumer memory for product information, its effects may persist over long periods of time.

Some limited consumer research has looked at the effects of ambient scent on memory (Morrin and Ratneshwar 2003; Smith, Standing, and De Man 1982). Most of these studies,

FIGURE 1

CONCEPTUAL FRAMEWORK FOR SCENT MEMORY RESEARCH

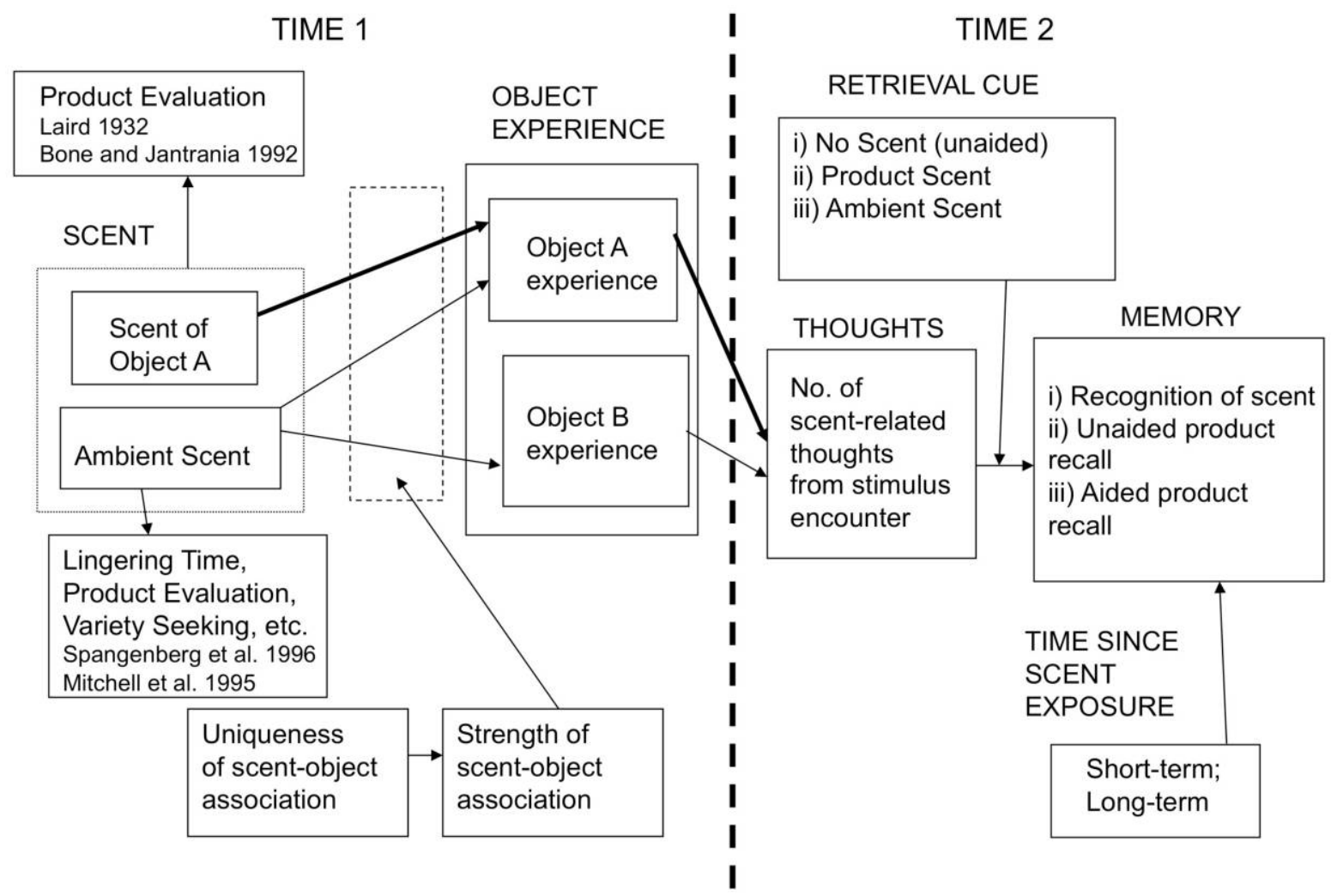


however, have also looked at contextual reinstatement effects, that is, whether the presence of scent at both the time of encoding and the time of retrieval improves memory (Smith et al. 1982), and they have utilized ambient scents rather than those associated with a single object in the environment (Morrin and Ratneshwar 2003; Smith et al. 1982). They typically focus on experimental participants being able to name objects that were seen by them rather than on recalling details about the objects (Morrin and Ratneshwar 2003). Finally, the effects are tested after relatively short delays (e.g., 5 minutes or 24 hours). In contrast, we focus on the effect of product (rather than ambient) scent on memory, we look at memory without reintroduction of the scent cue at the time of recall, we concentrate on memory for details about a product, and we examine memory for product information up to 2 weeks after product exposure. We next describe a framework that offers a rationale for why product scent may be particularly effective at improving memory for product information and thus is worthy of investigation.

\section{The Distinctiveness Hypothesis}

It is well established that distinctive stimuli encountered in the environment tend to attract attention (Green 1958) as well as greater encoding resources. Because objects that are perceptually distinctive are better encoded, they tend to be better remembered (Meyers-Levy and Tybout 1989). A stimulus can be distinctive either because it differs from its immediately surrounding context (i.e., primary distinctiveness) or because it is unexpected based on one's prior experiences and accumulated knowledge (i.e., secondary distinctiveness; Schmidt 1991).

Primary distinctiveness occurs when an object is perceived as being unusual in its surrounding context (Schmidt 1991). Thus, the features of a stimulus exhibiting primary distinctiveness do not match those it is surrounded by (e.g., encountering a word in red typeface in a list of words in black typeface; Schmidt 1991). We propose that products imbued with a scent will be well remembered because they are made distinctive within their surrounding context (i.e., exhibit primary distinctiveness) if other objects in the environment are not similarly scented.

Secondary distinctiveness occurs when an object is perceived as being unusual with respect to one's prior knowledge or with respect to information stored in long-term memory (Schmidt 1991). Secondary distinctiveness might occur if an unusual or unexpected ambient scent is encountered, for example, ambient scent in an environment that is not normally scented, such as a dentist's office. Unexpected ambient scent thus may attract attention to the scent itself or to all of the objects encountered in the scented environment.

We propose that the critical factor affecting scent's effectiveness at enhancing product memory is the extent to which a scent enhances the distinctiveness of the objectto-be-remembered (i.e., the product), not the extent to which the scent itself is distinctive. It seems logical to assume that the degree to which a scent will enhance an object's contextual distinctiveness would be a function of the number of objects with which the scent is associated in a given context. Because ambient scent, by definition, is not uniquely associated with a single object in the environment, but rather its associations are diffused across many or all objects in the environment, its ability to create associations with and enhance retrieval for any one particular object within that environment will be diminished. The number of scent-based associations with a single target object created by ambient scent will be fewer than the number resulting from exposure to a product scent or one that is associated with only that single target object. Although ambient scent may have the capacity to collectively enhance memory for all the objects encountered in the scented environment (e.g., all products, store signage, lighting, salespersons, etc.), its particular effects on a single product may be less than that of product scent.

Based on the preceding discussion, we expect, more generally, that, if consumers are exposed to products imbued with a scent, memory for product information will be significantly enhanced. This process is a function of the extent to which the scent makes the product stand out contextually, that is, enhances its contextual distinctiveness. In figure 1, scent of object $\mathrm{A}$ is more directly linked with object $\mathrm{A}$ experience than with object $B$ experience, whereas ambient scent's associations are diffused about equally across both objects. Thus, uniqueness of scent-object association affects the strength of scent-object association-it moderates the scent-object experience relationship and hence affects the number of scent-related thoughts and memory for the product. We study the effect of product A's scent on unaided recall for product $\mathrm{A}$ both in the short term and in the long term. We also study the effect of product scent and ambient scent on unaided recall for product $A$ and product $B$ in the long term. Additionally, we examine how the number of scent-related thoughts mediates the effect of scent on memory. We begin with an overview of our two studies.

\section{OVERVIEW OF STUDIES}

If scent were the main attribute of a product (e.g., perfume or room deodorizer; Schmitt and Schultz 1995), our question of scent-based memory for product information would have a fairly narrow range of applications. As such, we do not investigate products whose central attribute is scent; instead, we focus on products for which scent is a secondary or optional product attribute and whose central attribute is typically something other than scent, such as writing capability for a pencil or softness for a facial tissue.

Two studies are reported. In study 1, we demonstrate that product scent (in pencils) significantly enhances memory for product information and that this effect persists over time. In study 2, we replicate the memory-enhancing effect of product scent for a different type of product (facial tissues) embedded among other products (moisturizer, pencil) and obtain process evidence. Further, we explore the relative effectiveness of product scent versus ambient scent at improving memory for product information. 


\section{STUDY 1: SCENTED PENCILS}

Study 1 compares memory for product information related to scented versus unscented pencils. The experiment was conducted in a city where scented pencils are not very common. A small field study conducted among five different stores in this city showed that only nine pencils were scented among a total of 44 available pencils (20.4\%). As such, a scented pencil may be quite distinctive in this environment. For robustness, we compare an unscented type of pencil to two types of scented pencils, one with a less and one with a more congruent or common (for pencils) scent.

\section{Pretest}

Product Categories. A pretest was conducted among 31 undergraduates, who received $\$ 5.00$ for participation. Participants first rated the importance of the attribute of scent (on a scale of $1=$ extremely unimportant to $7=$ extremely important) for nine different types of products: moisturizer, facial tissue, pencils, soap, marker pens, toothpaste, lingerie, perfume, and hand cream. Scent was rated as relatively less important for pencils ( $M=2.90$ on a 7-point scale), but it was not totally unimportant ( $p<.01$ for paired $t$-test of difference from $M=1$ ).

Scents. Participants were then provided with samples of several scents (these included a range of scent types, e.g., woody, floral, and food-based) and were asked to rate each in terms of how common it was perceived to be for pencils (from $1=$ extremely uncommon to $7=$ extremely common). The scents were presented in small, tightly capped containers containing an ink blotter strip onto which had been added three to four drops of the scent. Each bottle was labeled with a capital letter (A to F). The samples were sniffed, and each was evaluated on the scale. Scents were presented in two different orders, the second being the reverse of the first order. Respondents were instructed to smell coffee grounds between consecutive stimuli to refresh their scent palettes. This was done to minimize contamination from one scent to the next and is an accepted practice in the fragrance industry to clear the nasal passage. The most and least common scents for the pencil product were pine $(M=5.42)$ and tea tree $(M=2.55$; paired $t=7.52, d f=$ $30, p<.01)$, respectively, and these were chosen as the scent stimuli for study 1 .

\section{Sample and Design}

One hundred and fifty-one students participated in return for course credit. The study consisted of a 3 (product scent: none, common, uncommon) $\times 3$ (time delay: none, short, long) full factorial design. In the no product scent conditions, the pencil was not imbued with a scent, whereas in the product scent conditions, the pencil was imbued with either a pine (common) scent or a tea tree (uncommon) scent. In the no time delay condition, memory was tested immediately after exposure to the pencil product, whereas in the time delay conditions, memory was tested after either a 24-hour (short) delay or a 2-week (long) delay.

\section{Stimulus Materials}

In the no product scent condition, the product was presented in the form of an unscented natural wood pencil. To create samples of the scented pencils, scents were dripped onto the wooden pencils, which were then placed in airtight bags for 48 hours. This process left no permanent marks.

\section{Procedure}

Study 1 was conducted in a laboratory setting over a 2week period, during which participants took part in two phases. Phase 1 consisted of an encoding phase in which participants were exposed to information about a new product via a print advertisement and were provided a sample of the product. All participants saw the same ad. The ad did not contain any references to scent or smell (see the wording of the ad in the appendix). Phase 2 consisted of a retrieval phase during which memory for brand information was tested. Participants were not made aware during the encoding phase that their memories would later be tested; thus, the learning that occurred consisted of incidental learning, as normally occurs in consumer contexts. The two phases of the study were performed either in a single session (during a 1-hour period on day 1) or in two separate sessions (a half hour in session 1 and a half hour in session 2, 24 hours later; or a half hour in session 1 and a half hour in session 2, 2 weeks later).

Upon arrival at the laboratory for phase 1, participants were seated at desks in separate private cubicles to afford them privacy and to prevent them from observing other participants. A printed booklet informed them that they would be taking part in a new product development research study. All participants read these instructions: "A new line of pencils is currently in its final stages of development. However, before the product is launched officially onto the market, we would like you to take a look at its list of attributes and consider them carefully. A sample of the pencil is provided as well. Using the provided sample, you may view, feel, and smell the pencil in order to better evaluate it." A print advertisement contained the product's brand name as well as several advertising claims discussing the product's attributes.

In phase 2, the retrieval phase, held in the laboratory, participants completed a survey, which tested their memory for the information contained in the advertisement. Participants were asked to write down the brand name of the pencil they received as well as everything else they could remember about the product, including all the attributes they could recall to describe it. Finally, the participant's age and gender were recorded. 


\section{Results}

Age and gender were included as covariates in the analyses of variance, but only gender emerged significant for some analyses and was retained. Two independent trained coders, who were neutral and blind to the experimental conditions, coded the responses. An item of recall was coded as correctly recalled when the brand name was spelled correctly (or almost correctly) or when a brand attribute was correctly recalled. Agreement was more than 99\%. Any disagreement was resolved by discussion.

Unaided Recall. We conducted an ANOVA on unaided recall as a function of product scent condition, time delay, their interaction, and the gender covariate. There was a significant main effect of product scent $(F(2,141)=39.65$, $\left.p<.01, \eta^{2}=.36\right)$ and a significant main effect of time delay $\left(F(2,141)=11.58, p<.01, \eta^{2}=.14\right)$, qualified by a significant interaction of time delay and product scent $(F(4$, $\left.141)=32.99, p<.05, \eta^{2}=.08\right)$. Gender was not significant $(p>.30)$.

Memory for Scented versus Unscented Pencils. Follow-up contrast tests compared the memory for unscented, scented-common, and scented-uncommon pencils at different time delays from product exposure. With no time delay, unaided recall for the unscented pencil $(M=3.27)$ was significantly lower than that for the scented-common pencil $(M=4.71 ; F(1,141)=10.10, p<.01)$ but not compared to the scented-uncommon pencil $(M=3.56 ; F(1,141)=$ $0.42, p>.50)$. Recall for the scented-common pencil was significantly higher than for the scented-uncommon pencil $(F(1,141)=6.00, p<.05)$.

After the short time delay, recall for the unscented pencil ( $M=1.94)$ was significantly lower than for both the scentedcommon pencil $(M=4.83 ; F(1,141)=42.02, p<.01)$ and the scented-uncommon pencil $(M=3.83 ; F(1,141)=17.71$, $p<.01)$. There was also a significant difference between the scented-common pencil and the scented-uncommon pencil $(F(1,141)=5.20, p<.05)$. With a long time delay after product exposure, the unscented pencil $(M=0.87)$ had significantly lower recall than both the scented-common pencil $(M=3.62 ; F(1,141)=30.37, p<.01)$ and the scenteduncommon pencil $(M=3.27 ; F(1,141)=22.32, p<.01)$, but there was no significant difference any longer between the scented-common pencil and the scented-uncommon pencil $(p>.45)$.

Effect of Time Delay. Additional follow-up contrasts compared the effects of time delay on memory for unscented, scented-common, and scented-uncommon pencils. These tests show that, for the unscented pencil, recall declined significantly from the no delay condition $(M=3.27)$ to the short delay condition $(M=1.94 ; F(1,141)=8.99, p<.01)$ and from the short delay conditon to the long delay condition $(M=0.87 ; F(1,141)=4.69, p<.01)$. As one can see, recall after 2 weeks was about one-fourth $(26.6 \%)$ of the initial recall for the unscented pencil. Thus, there was rapid for- getting for the unscented pencils, exhibiting a classic type of forgetting curve with a steep fall-off soon after exposure.

For the pencils infused with a common scent, recall was not significantly different in the no delay conditon $(M=4.71)$ and the short delay condition $(M=4.83$; $F(1,141)=0.07, p>.75)$. There was, however, a significant decrease in recall between the short and long delay conditions $(M=3.62 ; F(1,141)=7.22, p<.01)$. Nevertheless, recall after 2 weeks was still about three-fourths $(76.8 \%)$ of the initial recall-that is, there was relatively little forgetting of product information over time (i.e., a flatter forgetting curve).

For the pencils infused with an uncommon scent, there was no significant decline in recall among the various time delay conditions-no difference between no delay $(M=$ 3.56) and short delay $(M=3.83 ; p>.60)$, between no delay and long delay $(M=3.27 ; p>.45)$, or between short delay and long delay $(p>.20)$. Recall after 2 weeks was $91.8 \%$ of the initial recall for the pencil with the uncommon scent. So again we see relatively little forgetting of product information over time (i.e., a flatter forgetting curve). Indeed, we see that, while the initial recall level for the uncommon scent was lower than that of the common scent (when recall was tested immediately after exposure), the information that is encoded with the uncommon scent may be more resistant to decay over time than that encoded with the common scent.

\section{Discussion}

This pattern of results is consistent with our expectation that product scent would enhance unaided recall of product information and that this effect would persist over time. The effects imply that the impact of product scent on memory is not just a function of contextual reinstatement (i.e., the presence of scent at both encoding and retrieval), as has been shown in prior research (Herz 1997). That is, the memory effect is evident here even though there is no reintroduction of scent-based retrieval cues, suggesting that the memory improvement is due to superior encoding of scentassociated information at the time of exposure. We found such effects highly persistent over time, extending the findings from prior research that had used only short time delays. The long-lasting pattern of scent recognition memory was also extended to free recall of information associated with a product scent at the time of encoding.

In the short term and the medium term, the common scent was more effective at enhancing recall. In the long term (i.e., after 2 weeks), however, both types of scents were equally effective. The information encoded with the uncommon scent was more resistant to decay over time. The amount of information recalled with the common scent declined significantly after a long time delay (to 76.8\%), whereas there was no significant decline over time in the amount of information recalled with the uncommon scent $(91.8 \%)$. From this pattern of results, we speculate that information may be initially easier to encode with a common scent but, of the information that has been encoded, that 
which has been encoded with the uncommon scent is stronger and more resistant to decay.

In this study, we find that both common and uncommon scents are effective in enhancing memory. But, as we mentioned at the start of the experiment, with regard to pencils, possessing a product scent may itself be rather uncommon and contrary to expectations, so that any type of product scenting may attract attention and enhance memory.

In the next study, we use a product-facial tissues - that is more commonly sold in a scented format, so that scent is not as unexpected, and for which product scent is a more important selling attribute (as suggested by pretest results). We thus expand the generalizability of our results. We also utilize a setting in which the target product (facial tissues) is encountered among other types of products (i.e., moisturizer, pencils), which is more reflective of real world settings.

\section{STUDY 2: SCENTED TISSUES VERSUS AMBIENT SCENT}

In study 2, we not only attempt to replicate effects from study 1 but also attempt to compare the effectiveness of product scent versus ambient scent at enhancing memory for product information. Importantly, we also obtain process evidence in the form of scent-related associations stored in long-term memory. If it is true that product scent is more effective than ambient scent at focusing the consumer's attention and encoding efforts on the target product, as we have argued, then this should be evident not only in improved recall of target product information but also in a larger number of product/scent-related associations stored in long-term memory.

\section{Pretest}

Twenty-one pretest participants were recruited from the same population as those who were to participate in study 2 (i.e., undergraduate students at the same university). They were paid $\$ 7.00$ to participate. We tested four different scents: two of these were floral in nature (rose and jasmine), another was fruity (strawberry), and another had both fruit and floral elements (orange blossom). Two drops of each scent were put onto cotton balls and placed in tightly sealed double-layer zip lock bags for 48 hours prior to the pretest.

Participants were tested one at a time. The four bags with the scented cotton balls were lined up on tables in a random order. Participants sniffed the four scents, one at a time, so that the next packet was sniffed only when the participant was done rating the previous scent. Between scents, participants were given coffee grounds to sniff to clear the nasal system. Participants were free to start with any scent and proceed to any scent (on the questionnaire, they recorded which scent they were evaluating, each of which was identified by a capital letter: A, B, C, or D). The participants rated how common it would be to encounter each scent for each of six different product categories: facial tissues, moisturizer, room deodorizer, laundry soap, sunscreen, and sham- poo product (from $1=$ not at all common to $9=$ extremely common), how much they would like each of the products if it contained this scent $(1=$ not like at all to $9=$ like a lot), and how pleasant the scent would be for each of the products $(1=$ not at all pleasant to $9=$ extremely pleasant $)$. Respondents also rated how important scent was for each of the six products considered $(1=$ extremely unimportant to 7 = extremely important).

Analysis of the results suggested that two of the four scents, jasmine and orange blossom, were similarly rated for facial tissues and deodorizers. Specifically, orange blossom was rated as equally congruent (paired $t(20)=0.90$, $p>.35$ ), equally liked (paired $t(20)=0.47, p>.60$ ), and equally pleasant (paired $t(20)=1.02, p>.30$ ) for the facial tissues and room deodorizer product categories. Jasmine was also rated as equally congruent (paired $t(20)=0.57, p>$ .55 ), equally liked (paired $t(20)=1.01, p>.30$ ), and equally pleasant (paired $t(d f=20)=0.64, p>.50$ ) for the facial tissues and room deodorizer product categories. We chose to use orange blossom for study 2 due to the higher overall evaluation scores for this scent versus jasmine (mean liking of orange blossom $=4.20$ vs. 2.78 for jasmine, $p<.01$; mean pleasantness of orange blossom $=4.33 \mathrm{vs}$. 2.94 for jasmine; $p<.05)$. Scent was also considered a moderately important attribute for tissues $(M=4.86$ on a scale of $1-7)$, which is what we wanted in our study 2 to enhance the generalizability of our findings. Hence, tissues were chosen as the test product and orange blossom as the scent. Pencils and moisturizers were chosen as the other two products to be used in the study (distracter products) since they were very different from tissues and the three products would therefore be quite distinct.

\section{Sample and Design}

One hundred and fifty-three undergraduate students participated and were paid \$14 each. Two students stated that they had participated in a scent-related experiment before, and they were eliminated. In the second, or retrieval, phase of this study, eight students did not show up and thus had incomplete data, resulting in a final sample size of 143 . The no-shows were about evenly distributed among the scent conditions.

The study was a 3 (product: pencil, facial tissue, moisturizer) $\times 2$ (facial tissue product imbued with scent: yes, no) $\times 2$ (ambient scent in room: yes, no) mixed design, with the first factor manipulated within subjects and the next two factors manipulated between subjects. Thus, in phase 1, participants encountered either $(a)$ scented facial tissues in a scented room, $(b)$ unscented facial tissues in a scented room, $(c)$ scented facial tissues in an unscented room, or $(d)$ unscented facial tissues in an unscented room. Each participant was given the target product of facial tissues plus two other products (pencil, moisturizer), one at a time, during the first phase of the study. Facial tissues were always received second in the series of products evaluated to avoid primacy and recency effects. Whether pencil or moisturizer was first or last was counterbalanced by having half of the 
participants see the pencil first and the remaining half see the moisturizer first.

\section{Stimulus Materials}

In the ambient scent conditions, scent was emitted into the room for precisely 30 minutes before the experiment. For the product scent conditions, the facial tissue product was imbued with an equal number of drops of scent and placed into tightly sealed double-layer zip lock bags for 48 hours prior to the experiment commencing (note: the pencil and the moisturizer products were never imbued with scent).

\section{Procedure}

As in study 1, participants took part in two phases. In the first (encoding) phase, they were exposed to the stimuli, and in the second (retrieval) phase, they were asked to freely recall all they could about the stimuli. We chose to test memory performance 2 weeks after stimulus exposure, as we were most interested in the persistent effects of scent on long-term memory.

Encoding Phase. In phase 1, the encoding phase, we had five experimenters attending to 12-16 participants at a time (i.e., we ran sessions consisting of $12-16$ persons at a time). Participants were presented with the three products (moisturizer, facial tissues, and pencils), one at a time (see the appendix). Upon arrival at the laboratory for phase 1, participants were seated at desks, separated at least 5 feet or more away from each other. A printed booklet informed participants that they would be taking part in a new product development research study. They read these instructions:

\footnotetext{
You will be assessing a [moisturizer, facial tissue, pencil] product in its final stages of development. We would like you to take a look at its list of attributes and consider them carefully. A sample of each will be provided as well. Using the provided sample, you may examine each product in order to better evaluate it. When you have finished testing one product, please stop, put up your hand and you will be given the next product.
}

For each product, a print advertisement that contained the product's brand name as well as several advertising claims discussing the product's attributes was provided. Each print ad contained 10 copy points, none of which referred to product scent. Participants in this study were also never explicitly instructed to smell any of the products.

Experimenters recorded the amount of time each participant spent assessing each product. The amount of time spent with each product was recorded from the time they were given the product until they requested the next product. Participants examined each product with its accompanying print advertisement, evaluated the product on three closedended items (product quality, effectiveness, liking of product - extremely low/extremely high; 1-9 scales), and completed a thought-listing task, where they were asked to write down everything that went through their minds while examining the product. At the end of being exposed to all three products, participants completed several 9-point, closedended items, which make up the pleasure and arousal dimensions of Mehrabian and Russell's (1974) PAD scale (unhappy/happy, unsatisfied/satisfied, annoyed/pleased, bored/ relaxed, depressed/contented, relaxed/stimulated, calm/excited, sleepy/wide awake, unaroused/aroused). They also completed Fisher's (1974) scale of affective response to the environment (tense/relaxed, uncomfortable/comfortable, depressing/cheerful, drab/colorful, boring/stimulating, unlively/lively, dull/bright, uninteresting/interesting).

Retrieval Phase. Upon returning to the laboratory for phase 2, participants' memories were tested for each product in the order in which they had earlier been supplied the products during the encoding phase (which was counterbalanced across participants). They were also asked some other questions, as described below. Unaided recall was measured by asking participants to fill in the brand name of the first product they had examined at time 1; they were provided with the category name (e.g., moisturizer). Then they were asked to remember as much else as they could about that product. More specifically, they were asked to "write down everything you can remember about the [body moisturizer, facial tissues, pencil]. Please INCLUDE all the attributes you can remember to describe the [body moisturizer, facial tissues, pencil]." Then the participants were asked to evaluate the product on the same closed-ended items that were used in phase 1 (quality, effectiveness, liking). This process was repeated two more times for each of the remaining products until unaided recall and product evaluations for all three products had been assessed. Recall measures were coded similarly to those in study 1 , and agreement between the two coders was 95\%, with disagreements resolved by discussion.

After completing the recall measures, participants responded to the item, "How congruent would it be for this type of scent to be used in _ [each of the three products]?" on a 9 -point scale $(1=$ not at all, $9=$ very much); answered an open-ended probe for what they believed the purpose of the study to be; and reported their age and gender. Analysis of the open-ended probe showed that 15 participants mentioned something related to scent, but none of them guessed that the study was about the effect of scent on memory, and so none were eliminated from the sample on the basis of hypothesis guessing. Neither age nor gender was significant in this study ( $p$ 's $>0.2$ ) and are not included in the analyses.

\section{Results}

Scent Awareness. Five percent of participants thought the room was scented when the room was not ambiently scented, compared to $27 \%$ in the condition where the room was ambiently scented $\left(\chi^{2}(1)=13.46, p<.01\right)$. Thus, participants were more likely to report the room was scented when it was, but the overall awareness level of room scenting was low. We also checked whether awareness of the ambient scent was moderated by product scenting, and it 
was not, with awareness of ambient scent about the same whether the tissue was scented or not (23\% vs. $31 \%$ aware of ambient scent, $\left.\chi^{2}(1)=4.33, p<.05\right)$. Also, participants were more likely to report that the product was scented when it was versus was not $\left(69 \%\right.$ vs. $26 \% ; \chi^{2}(1)=27.94, p<$ $.01)$. We checked whether ambient scenting affected this pattern of results, and it did not. When the tissue product was scented, about an equal proportion of participants noticed that the tissue was scented in the unscented room as in the scented room $\left(64 \%\right.$ vs. $\left.74 \% ; \chi^{2}(1)=2.64, p>.10\right)$.

Main Results. Since each participant in this study provides responses for three distinct products, we analyze the data with multivariate analyses of variance. A MANOVA can accommodate correlations among the dependent measures as well as control for the overall Type I error rate (Bray and Maxwell 1985). Since we include covariates in the analyses more specifically, we use MANCOVA. Each MANCOVA includes three dependent variables, representing measures from the three products examined by each participant (moisturizer, facial tissue, pencils), two independent variables: the between-subjects factors of ambient scent (yes/no) and product scent (yes/no), their interaction, as well as covariates for product order and perceived scent congruence for each of the three products. Like an ANOVA, where significant omnibus $F$-tests are followed up with mean comparisons, when omnibus multivariate $F$-tests are found significant from the MANCOVA, we examine the results of the univariate $F$-tests from the MANCOVA to see which of the three dependent measures are affected by the independent variables and covariates (Bray and Maxwell 1985).

Unaided Recall. Similar to the procedure for study 1, we calculated a correct unaided recall score for each participant based on the total number of attributes correctly recalled plus the brand name if correctly recalled (total possible correct recall was 11 ; see table 1 for means). Analysis of the multivariate $F$-tests from the MANCOVA showed that the only effect that was statistically significant was product scent $\left(F(3,133)=2.741, p<.05, \eta^{2}=.06\right)$. Neither ambient scent $(p>.75)$ nor its interaction with product scent $(p>.75)$ was significant, nor were any of the covariates $(p$ 's $>.25)$.

The univariate $F$-tests showed that product scent had a significant impact on unaided recall for the tissue product $\left(F(1,135)=5.111, p<.05, \eta^{2}=.05\right)$ but not for the moisturizer or pencil products $(p$ 's $>.85)$. The mean number of items recalled about the tissue product increased from 4.65 to 5.28 when this product was scented.

This result replicates that of study 1 , where we found a product with scent (vs. without) resulted in greater unaided recall, especially after a time delay. Here we find the same result using a different product category (tissues rather than pencils) encountered within a multiple-product setting, with memory tested after a 2-week time delay. We found no facilitative effect of ambient scent on unaided recall, supporting the idea that product scent is more effective at enhancing memory for information about a single target in the environment.
TABLE 1

STUDY 2: UNAIDED RECALL

\begin{tabular}{lccc}
\hline \hline & $\begin{array}{c}\text { Tissue } \\
\text { product } \\
\text { scent } \\
\text { (no) }\end{array}$ & $\begin{array}{c}\text { Tissue } \\
\text { product } \\
\text { scent } \\
\text { (yes) }\end{array}$ & \\
Product & & & p-value \\
\hline Facial tissue: & 4.75 & 5.44 & \\
$\quad$ Ambient scent (no) & 4.55 & 5.12 & .07 \\
$\quad$ Ambient scent (yes) & 4.65 & 5.28 & .15 \\
$\quad$ Total & & & .02 \\
Moisturizer: & 5.02 & 4.95 & .85 \\
$\quad$ Ambient scent (no) & 4.79 & 4.80 & .97 \\
Ambient scent (yes) & 4.91 & 4.88 & .91 \\
$\quad$ Total & & & \\
Pencil: & 4.70 & 4.46 & .51 \\
$\quad$ Ambient scent (no) & 4.26 & 4.41 & .68 \\
$\quad$ Ambient scent (yes) & 4.48 & 4.44 & .86 \\
$\quad$ Total & & & \\
\hline
\end{tabular}

We have suggested that the reason that product scent may be especially capable of enhancing product memory is because it enhances the product's distinctiveness. This process should create strong associations in long-term memory between a single target, that is, the specific product that has been imbued with a scent, and the scent itself. The scentbased associations created as a result of ambient scent, in contrast, may be diffused across all the elements encountered in the environment (multiple products, people, events, room characteristics, etc.). To test this idea further, we conduct a mediation analysis on unaided recall as a function of scentrelated mentions generated during the product information retrieval phase.

Since none of the advertisement information referred to scent, the only source of such associations, if they were created and stored in long-term memory, would have been the presence or absence of scent itself (either in the product or in the surrounding atmosphere). These scent-related mentions, which are measured 2 weeks after product exposure, suggest that such associations, if they exist, tend to persist in long-term memory.

Mediation Analysis. We conducted a mediation analysis using scent-related thoughts mentioned during the retrieval phase (Baron and Kenny 1986), that is, when we asked participants to recall everything they could about the product in the unaided recall task. Using ANOVAs, we first regressed unaided recall for the tissue product on the independent variable, product scent, along with the covariates. The only significant effect was product scent $(F(1,137)=5.08, p<.05)$. Then we regressed the mediator, scent mentions, on the independent variable and the covariates. The only significant effect was product scent $(F(1,137)=31.87, p<.01)$. Then we regressed the dependent variable of unaided recall for facial tissues on product scent, scent mentions, and the covariates. The mediator was statistically significant $(F(1,136)=5.89, p<$ $.05)$, whereas the independent variable was not $(F(1,136)=$ 
1.02, $p>.30)$, suggesting full mediation. A Sobel (1982) test of the impact of the mediator was significant $(t=2.22, p<$ $.05)$.

\section{Ruling Out Alternative Process Explanations}

We measured several additional cognitive, affective, and behavioral process measures, which we report below, to explore other potential alternative explanations for the effect of scent on memory.

\section{Cognitive Processing during the Encoding Phase.}

During the encoding phase, we asked participants to write down all their thoughts after evaluating each product. On average, participants listed 3.9 thoughts about the moisturizer, 4.5 thoughts about the tissue, and 3.6 thoughts about the pencil. A MANCOVA conducted on the number of thoughts listed after seeing each of the products suggests that none of the effects were significant ( $p$ 's $>.10)$ except for the covariate of product order $(F(3,133)=4.42, p<$ $.01)$. The univariate $F$-tests showed that product order had an impact on the number of thoughts only for the pencil product $(F(1,135)=5.69, p<.05)$. A follow-up ANOVA with product order entered as a factor showed that more thoughts were listed about the pencil when it came last in the set of products $(M=3.86)$ rather than first $(M=$ 3.26), suggesting a recency effect. The results do not suggest that either product scent or ambient scent affects the overall amount of cognitive processing engaged in by consumers or that amount of processing mediates the effects of scent on memory.

Scent-Related Thoughts during the Encoding Phase. We also conducted a MANCOVA more specifically on the number of scent-related thoughts mentioned during the encoding phase. The multivariate $F$-tests showed that product scent was significant $\left(F(3,133)=15.15, p<.01, \eta^{2}=\right.$ $.25)$, as was product order $\left(F(3,133)=3.06, p<.05, \eta^{2}\right.$ $=.06)$, indicating recency effects. The univariate $F$-tests showed that product scent had a significant effect for the tissue product $(F(1,135)=45.84, p<.01)$ but not for the moisturizer $(p>.30)$ or pencil $(p>.80)$ products, as we would expect. When the facial tissue product was imbued with a scent, participants listed more scent-related thoughts at the time of encoding ( $M=0.37$ vs. $M=1.02 ; F(1,132)$ $=46.88, p<.01)$.

The number of scent-related thoughts listed at the time of encoding for each of the products, however, did not mediate the effects of scent on unaided recall in the retrieval phase. This result suggests that it is not necessarily the sheer number of scent-related thoughts generated at the time of encoding that mediates the effects of scent on long-term memory but rather the persistency of such scent-related thoughts that last over time and are present at the time of retrieval (in this case, as much as 2 weeks from the time of exposure).

Affective Responses. Neither pleasure (happy, satisfied, pleased, relaxed, contented; Cronbach's $\alpha=.91$ ) nor arousal (stimulated, excited, aroused; coefficient; $\alpha=.80$ ) were sig- nificantly affected by product scent $(p>90)$, ambient scent $(p>.65)$, their interaction $(p>.60)$, or any of the covariates ( $p$ 's $>.15)$. Thus, consumers' mood and arousal levels were not affected by the presence of either ambient scent or product scent. This result is in accord with the findings of Bone and Ellen (1999), who found in their meta-analysis that only $16.1 \%$ of the tests of the effect of scent on mood or arousal were statistically significant. We similarly found no significant effects of scent condition on consumers' affective response to the environment (Fisher's scale [1974]; $\alpha=$ $.93, p$ 's $>.05)$.

Product Evaluation. While it was not the focus of this research, we also assessed the impact of scent on product evaluations both at the time of encoding and at the time of retrieval (alphas for the three products at the time of encoding ranged from .87 to .89 ; alphas at the time of retrieval ranged from .91 and .93). We found no significant effects of ambient scent or product scent on product evaluation either at the encoding or the retrieval phase or as a difference between the two phases ( $p$ 's $>0.10$ ).

Time Spent with Products. We conducted a MANCOVA on the amount of time spent with each of the three products during the encoding phase of the experiment. The multivariate $F$-tests showed that both ambient scent $(F(3$, $\left.133)=10.75, p<.01, \eta^{2}=.19\right)$ and product scent $(F(3$, $\left.133)=3.80, p<.05, \eta^{2}=.08\right)$ had a significant impact on time spent, as did product order $(F(1,133)=19.54, p<$ $\left..01, \eta^{2}=.31\right)$, which indicated recency effects. The univariate $F$-tests showed that ambient scent significantly affected time spent with the pencil product $(F(1,135)=17.43, p<$ $.01, \eta^{2}=.11$ ), decreasing it from a mean of 6.7 to a mean of 5.4 minutes when the room was scented. The univariate $F$-tests also showed that product scenting increased time spent with the tissue (from 4.89 to 5.80 minutes; $F(1,135)=$ $\left.10.60, p<.01, \eta^{2}=.07\right)$, as well as time spent with the moisturizer (from 6.1 minutes to 6.7 minutes; $F(1,135)=$ 5.89, $\left.p<.05, \eta^{2}=.04\right)$. The increased time spent with the tissue product is consistent with our contention that product scent enhances the distinctiveness of the product and thus results in greater attention paid to the product. The effect on moisturizer is not clear. As with the other process measures reported in this section, however, time spent with the products did not mediate the effects of scent on memory performance. Thus, we conclude that none of the possible alternative process measures adequately account for the effects of scent on memory.

\section{Discussion}

In this study, we replicate the memory-enhancing effect of product scent using a different type of product (facial tissues) that is encountered within a set of other products, as might occur in a store setting. We found that, when a product is scented, long-term memory for that product's other (i.e., non-scent-related) attributes increases. We also found that product scent was more effective than ambient 
scent at enhancing memory for product-related information, which is consistent with the distinctiveness hypothesis. We showed that product scent creates strong, longlasting associations between the scent and product attribute information, with these associations accessible as much as 2 weeks after the time of exposure. We did not find that scent's memory-enhancing effect is due to such other processes as cognitive elaboration, mood, arousal, environmental affect, or time spent with the products.

Although ambient scent did not similarly enhance memory for a single target in the environment, ambient scent may have the capacity to enhance memory for a collection of objects encountered within an environment, since its effects may be diffused across all such objects. Thus, for example, Morrin and Ratneshwar (2003, study 2) found that, when they exposed participants to 42 different brands in the presence of ambient scent, the proportion recalled increased from $13 \%$ to $15 \%$. However, it is unlikely that memory for a single brand encountered in such an environment would be measurably altered by the diffused effects of ambient scent.

What we have shown here is that product scent is highly effective at enhancing memory for information about a single target product. We have offered one possible rationale for its efficacy, that is, the number of scentrelated associations created in long-term memory that are focused on that product.

\section{GENERAL DISCUSSION}

Interest in sensory marketing appears to be on the rise, especially in previously neglected areas such as olfaction and touch (Peck and Childers 2008). In this exploratory study, we focus on olfaction, or the sense of smell, and more specifically, on the significant effects that product scents can have on memory for product information.

We demonstrate that product scent significantly enhances recall of product information and that this enhanced memory for product information persists over time-for at least 2 weeks after the time of exposure. We further find that product scent creates long-lasting scentproduct associations in memory. We found that product scent enhanced memory for product information more effectively than did ambient scent, whose effects may be diffused across numerous objects in the environment. While our results are consistent with the distinctiveness hypothesis, they do not rule out all alternative explanations. For example, it is possible that the level of intensity of the ambient scent used in the present research was below that necessary for significant memory effects to emerge. It is also possible that the ambient scent improved memory for several items encountered in the environment and if added together would demonstrate collective improvement in memory performance.

Nevertheless, we suggest that it is the extent to which a scent makes the object(s) to be remembered distinctive that determines its effectiveness at enhancing memory for those objects. If interest is on the extent to which scent enhances memory for information about a specific product, the preliminary evidence presented here suggests that the scent needs to be associated with just that single object (rather than diffused or shared across multiple objects in the environment). In this way, the target product's contextual distinctiveness is enhanced, encoding resources are focused, and subsequent retrieval is improved.

Unlike most prior studies examining the effects of scent on memory, we did not look at the effects of scent-cueaided performance (Herz 1997). Instead, we looked at unaided recall, which may be more important not only for theoretical reasons but for managerial reasons as well. If people remember a product and its attributes at the time of purchase many weeks after being exposed to it (in a shop, in a hotel, or at a friend's house), then they can look for it during their shopping trip and purchase it. In the real world, the scent cue as an aid for recall will work only if one picks up the product and the packaging is permeated with a scent (or the product scent seeps through the packaging).

Of course, this is an exploratory effort, and additional research is needed to test whether the proposed distinctiveness hypothesis indeed explains the degree to which scent type (ambient vs. object-specific) enhances the distinctiveness of the object to be recalled. Future research could also study whether the effects of product scent are diminished when competitive products are similarly scented. Our distinctiveness hypothesis would suggest that they would be.

In the United States, legally, scents may be trademarked if they serve to identify the product source or brand name, even if they do not serve a functional purpose. However, there have been relatively few scentbased trademarks established. This may be the case because until now people have not understood scent's strong ability to enhance product identification. As such, we hope that this research not only shows how important scent can be in enhancing memory for product information but also contributes to building arguments for why olfactory trademarks should be considered.

\section{APPENDIX}

\section{ADVERTISING CLAIMS PRESENTED}

Writzwell Pencil:

- Is endorsed with the Green Seal environment standard

- Contains superior graphite lead

- Are made from premium oak trees that hail from California

- Are non toxic and acid free

- Offer a smooth writing process for all users' styles

- Are perfectly suited for art work such as sketching

- Require 45 percent less sharpening on average

- Come only in 3 sizes - 6 inch, 4 inch, and 2 inch

- Produce writing lines that do not smear easily

- Feature a smooth finishing for easy gripping 
Clarisilkia Moisturizer (presented only in study 2):

- Comes in handy tubes of different sizes

- Is a non-greasy cream that absorbs easily into the skin

- Leaves your skin feeling soft and healthy

- Is formulated with Aloe Vera and other natural plant extracts

- Contains Vitamin $\mathrm{C}$ and $\mathrm{E}$ to fight aging

- Is great for people whose skin peels often

- Eliminates discoloration of the skin

- Contains sunscreens to give great protection against the sun

- Is suitable for all skin types

- Is made entirely with Swiss Pride

Ceville Facial Tissues (presented only in study 2):

- Contains a mild antiseptic formula to help eliminate the spread of bacteria

- Made of softer fibers so it's gentler on your skin

- Is available in a wide variety of colors

- Comes packaged in handy portable package sizes

- Triple-ply thickness for enhanced strength

- Is great for people whose skin is sensitive

- Is available embossed with attractive designs

- Made of paper with minimal lint, to maximize cleanliness

- Tested for high absorbency

- Made in France

\section{REFERENCES}

Baron, Reuben M. and David A. Kenny (1986), "The Moderator Mediator Variable Distinction in Social Psychological-Research: Conceptual, Strategic, and Statistical Considerations," Journal of Personality and Social Psychology, 51 (6), 1173-82.

Bird, Steve (2000), "Game, Scent and Match for Grassy Tennis Balls," The Times (London), May 25, Home News Section.

Bone, Paula Fitzgerald and Pam Scholder Ellen (1999), "Scents in the Marketplace: Explaining a Fraction of Olfaction," Journal of Retailing, 75 (2), 243-62.

Bone, Paula Fitzgerald and Swati Jantrania (1992), "Olfaction as a Cue for Product Quality," Marketing Letters, 3 (July), 289-96.

Bosmans, Anick (2006), "Scents and Sensibility: When Do (In)Congruent Ambient Scents Influence Product Evaluations?" Journal of Marketing, 70 (3), 32-43.

Bray, James H. and Scott E. Maxwell (1985), Multivariate Analysis of Variance: Quantitative Applications in the Social Sciences, Thousand Oaks, CA: Sage.

Brookman, Adam L. (1999), Trademark Law: Protection, Enforcement and Licensing, New York: Aspen. (Page 212 in 2006 supplement, citing Clarke [1990] 17 USPQ2d 1238 [TTAB 1990].)

Buck, Linda and Richard Axel (1991), "A Novel Multigene Family May Encode Odorant Receptors: A Molecular Basis for Odor Recognition," Cell, 65 (1), 175-87.

Carter, Meg (1995), “This Isn't Just Any Old Tyre: Sniff It and See Why . . . ," Independent (London), October 3, Consuming Section, 11.

Engen, Trygg, James E. Kuisma, and Peter D. Eimas (1973), "Short-Term Memory of Odors," Journal of Experimental Psychology, 99 (2), 222-25.
Engen, Trygg and Bruce M. Ross (1973), "Long-Term-Memory of Odors With and Without Verbal Descriptions," Journal of Experimental Psychology, 100 (2), 221-27.

Fisher, Jeffrey (1974), "Situation-Specific Variables as Determinants of Perceived Environmental Esthetic Quality and Perceived Crowdedness," Journal of Research in Personality, 8 (August), 177-88.

Green, Robert T. (1958), "The Attention-Getting Value of Structural-Change," British Journal of Psychology, 49 (4), 311-14.

Herz, Rachel S. (1997), "The Effects of Cue Distinctiveness on Odor-Based Context-Dependent Memory," Memory and Cognition, 25 (3), 375-80.

Laird, Donald A. (1932), "How the Consumer Estimates Quality by Subconscious Sensory Impressions: With Special Reference to the Role of Smell," Journal of Applied Psychology, 16 (3), 241-46.

Mehrabian, Albert and James A. Russell (1974), An Approach to Environmental Psychology, Cambridge, MA: MIT Press.

Meyers-Levy, Joan and Alice M. Tybout (1989), "Schema Congruity as a Basis for Product Evaluation," Journal of Consumer Research, 16 (1), 39-54.

Mitchell, Deborah J., Barbara E. Kahn, and Susan C. Knasko (1995), "There's Something in the Air: Effects of Congruent or Incongruent Ambient Odor on Consumer Decision Making," Journal of Consumer Research, 22 (September), 229-38.

Morrin, Maureen and Srinivasan Ratneshwar (2000), "The Impact of Ambient Scent on Evaluation, Attention, and Memory for Familiar and Unfamiliar Brands," Journal of Business Research, 49 (2), 157-65.

- (2003), "Does It Make Sense to Use Scents to Enhance Brand Memory?" Journal of Marketing Research, 40 (February), 10-25.

Peck, Joann and Terry L. Childers (2008), "If It Tastes, Smells, Sounds, and Feels Like a Duck, Then It Must Be a . . . : Effects of Sensory Factors on Consumer Behaviors," in Handbook of Consumer Psychology, ed. Curtis P. Haugtvedt, Paul M. Herr, and Frank R. Kardes, New York: Psychology, 193-219.

Peterson, Lloyd R. and Margaret Jean Peterson (1959), "ShortTerm Retention of Individual Verbal Items," Journal of Experimental Psychology, 58, 193-98.

Schmidt, Stephen R. (1991), "Can We Have a Distinctive Theory of Memory?" Memory and Cognition, 19 (6), 523-42.

Schmitt, Bernd H. and Clifford J. Schultz II (1995), "Situational Effects on Brand Preferences for Image Products," Psychology and Marketing, 12 (5), 433-46.

Shepard, Roger N. (1967), "Recognition Memory for Words, Sentences and Pictures," Journal of Verbal Learning and Verbal Behavior, 6 (1), 156-63.

Smith, David G., Lionel Standing, and Anton DeMan (1982), "Verbal Memory Elicited by Ambient Odour," Perceptual and Motor Skills, 74 (2), 339-43.

Sobel, Michael E. (1982), "Asymptotic Confidence Intervals for Indirect Effects in Structural Equation Models," in Sociological Methodology, ed. Samuel Leinhardt, Washington, DC: American Sociological Association, 290-312.

Spangenberg, Eric R., Ayn E. Crowley, and Pamela W. Henderson (1996), "Improving the Store Environment: Do Olfactory Cues Affect Evaluations and Behavior?" Journal of Marketing, 60 (April), 67-80.

Zucco, Gesualdo M. (2003), "Anomalies in Cognition: Olfactory Memory," European Psychologist, 8 (2), 77-86. 